\title{
Visions in a Crystal Ball: The Future of Peritoneal Dialysis
}

\author{
Joanne M. Bargman Michael Girsberger \\ Division of Nephrology, University Health Network Toronto General Hospital, Toronto, ON, Canada
}

\section{Keywords}

Future of peritoneal dialysis - Cardiorenal syndrome .

Hyperbranched polyglycerol · Incremental peritoneal dialysis - Wearable medical devices - Peritoneal dialysis in acute kidney injury $\cdot$ Home generation of peritoneal dialysis solutions

\section{Abstract \\ Background: Peritoneal dialysis (PD) is one of the corner stones of renal replacement therapy and should be strongly considered if preemptive kidney transplantation is not avail- able. Summary: There are several initiatives that may help the growth in the use of PD around the world. First, PD is an underused and valuable option in patients with heart failure and the chronic cardiorenal syndrome, especially in those with frequent hospitalizations despite optimal medical ther- apy. To identify these patients, an interdisciplinary approach of nephrologists and cardiologists is needed. These patients and other CKD patients with significant residual kidney function may do well with a regimen employing fewer than the usual number of bag exchanges, referred to as "incre- mental" dialysis. Second, acute kidney injury (AKI) is a world- wide burden with high morbidity and mortality, especially in low income countries. To reach the goal of zero prevent-}

\section{KARGER}

() 2018 S. Karger AG, Basel

E-Mail karger@karger.com

www.karger.com/bpu able deaths caused by AKI by 2025 endorsed by the International Society of Nephrology, PD is the therapy of choice for treatment in this setting. Third, although dextrose has served well as the osmotic agent in PD solutions, there has been a continuous search for alternative agents. Hyperbranched polyglycerol might be such an osmole. Finally, to obviate the need for production and delivery of bags of PD solution, the development of home-generated dialysate is of interest. Key Message: The future of PD lies not only in accruing experience from the past decades, but also in staying open to other uses.

Video Journal Club 'Cappuccino with Claudio Ronco' at http://www.karger.com/?doi=485158. @ 2018 S. Karger AG, Basel

\section{Introduction}

"The best way to predict the future is to create it." - Abraham Lincoln

Since its first experimental use in the 19th century [1], peritoneal dialysis (PD) has become a corner stone of renal replacement therapy (RRT). For the future of PD, it is important to elaborate what is needed to improve current practice. 
Fig. 1. Pathophysiology of the cardio-renal syndrome. The downstream effects of diminished cardiac output and the vicious cycle leading to the cardio-renal syndrome and diuretic-resistant heart failure.

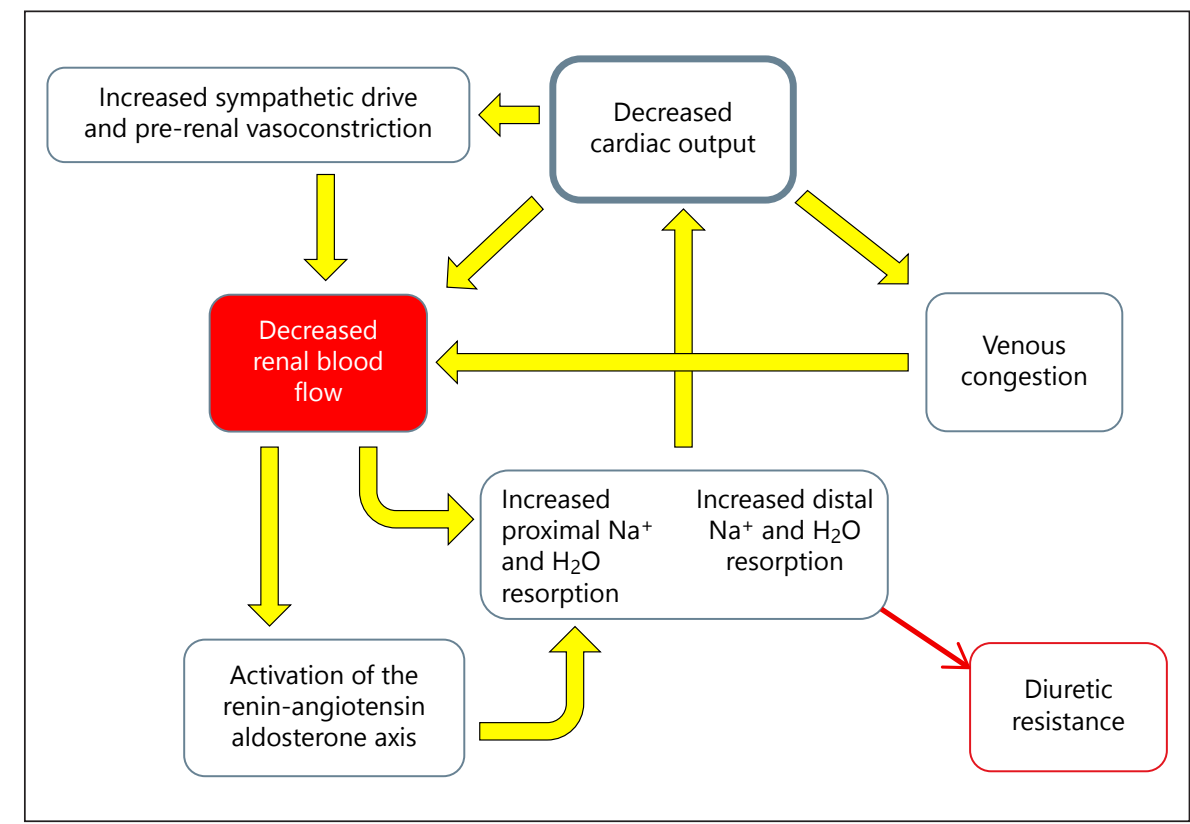

\section{PD in Chronic Heart Failure}

Heart failure (HF) is a major cause of hospitalizations, health care costs, morbidity and mortality. Due to the higher incidence of HF with age and the growing proportion of elderly people, the number of patients with HF is expected to rise. A significant percentage of patients with HF also have reduced kidney function, whereby a decline in kidney function can worsen HF and vice versa. This maladaptive interaction of the kidney and the heart led to the term cardio-renal syndrome (CRS) entailing 4 distinctive clinical scenarios: acute or chronic HF leading to kidney failure (CRS I and II, respectively) and acute or chronic kidney failure leading to HF (CRS III and IV, respectively). While renal replacement therapies in patients with CRS III and IV are well established, the role of such therapies in CRS I and II is less clear.

In CRS I, acute HF leads to a significant reduction in kidney function. Decreased cardiac output and the resultant decrease in renal blood flow result in increased proximal and distal tubular sodium and water retention, further compromising cardiac function. This vicious cycle is aggravated by an increased sympathetic drive and activation of the renin-angiotensin-aldosterone axis triggered by low effective circulating volume. An additional aspect is venous congestion called backward failure as a consequence of reduced cardiac function, probably one of the most important hemodynamic factors in CRS [2] (Fig. 1). Overall, these maladaptive responses lead to a gradual de- cline in glomerular filtration rate with gradual shrinking of kidney mass and eventually irreversible fibrosis if kidney hypoperfusion is sustained (CRS II).

The rationale of using continuous ultrafiltration through PD compared to medical therapy includes obviating the side effects of diuretics such as activation of the renin-angiotensin- and the sympathetic nervous system. Continuous fluid removal also has a reduced risk of hypotension with its deleterious effects on organ function, which makes it more tolerable in hypotensive patients. Additionally, the removal of proinflammatory factors through PD might improve cardiac function [3]. An important consideration when comparing PD to intermittent hemodialysis in this setting is the absence of myocardial stunning in $\mathrm{PD}$, a well-documented phenomenon in hemodialysis associated with higher mortality $[4,5]$. Another advantage of PD compared to HD includes not needing an arteriovenous access with the risk of high flow fistulas increasing cardiac work and precipitating high output HF.

There are 2 patient groups with CRS who could benefit from PD: patients with non-end-stage kidney disease (ESKD) and those with ESKD.

In patients with CRS and non-ESKD, studies investigating $\mathrm{PD}$ as a potential therapeutic modality in refractory volume overload showed favorable outcomes. In a study by Courivaud et al. [6], left ventricular ejection fraction improved significantly after the initiation of PD. Furthermore, there was an impressive $90 \%$ reduction in hos- 
pitalization days after the initiation of PD. Other studies showed similar results $[7,8]$. Overall, PD-specific complications, such as peritonitis, were rare.

In contrast, studies in patients with CRS and ESKD showed less favorable results for PD. However, it is important to point out that the study design in these trials differed considerably. Given the fact that the patients had ESKD, PD was compared to another RRT, namely, HD, rather than comparing endpoints before and after the initiation of therapy.

In summary, there is building evidence that PD in CRS patients with refractory volume overload without ESKD might be a beneficial and feasible alternative to medical therapy alone. It is important to put the focus in these often severely ill patients more on quality of life rather than survival, and therefore, the reported reduction in hospitalizations would be a meaningful endpoint. Furthermore, PD regimens in patients without ESKD differ from a "full" dialysis dose, showing a benefit sometimes with only one hypertonic fill overnight without any daytime exchanges. The observation that reported PD complications were rare makes this modality an attractive alternative to medical treatment alone. The future goal is to identify those patients who might benefit from PD. There is a need for interdisciplinary collaboration of cardiologists and nephrologists to tackle this challenge.

\section{PD in Acute Kidney Injury}

PD was a widely accepted treatment for acute kidney injury (AKI) before the introduction of hemodialysis in the late 1970s and early 1980s. While it is nowadays rarely used in the developed world, it is still the therapy most commonly used for AKI in developing countries due to its low cost and simple technology. An interesting study [9] published in 2012 reported that $36 \%$ of participants at 3 major nephrology meetings considered PD an option for AKI, but only $15 \%$ actually used it in this setting. PD was more often used by practitioners from Asia than Europe or Northern America.

Advocating PD as a general therapy for AKI in the future for adult patients in developed countries is not practicable, given the availability of continuous hemodialysis and the fact that modality decisions in intensive care units are often made by intensivists or anesthesiologists and not nephrologists. Nevertheless, it is important to note that there are certain advantages to $\mathrm{PD}$, such as avoidance of vascular access and anticoagulation, car- diovascular stability, earlier recovery of renal function [10], and reduced risk of disequilibrium that could make it a valuable option in selected patients. Furthermore, several studies showed no difference in mortality comparing PD in AKI to extra corporal blood purification [10-12].

While AKI is a worldwide disorder with over 13 million people being affected each year and over 2 million dying as a result, the vast majority of AKI (85\%) occurs in middle- and low-income countries [13]. Therefore, the parts of the world that use PD in AKI seem to be affected the most. In 2013, the International Society of Nephrology addressed this problem by launching the ambitious 0 by 25 initiative with the goal of eradicating deaths due to AKI by 2025. After a first initial step of undertaking a cohort study [14] showing similarities in risk factors and causes of AKI across all countries, the International Society of Nephrology is currently carrying out projects in Africa, Asia and Latin America to reduce morbidity and mortality from AKI in middle- and lowincome countries.

In summary, the worldwide burden of AKI is high with an unacceptable disproportion in developing countries, making a global effort to ameliorate this disparity essential in the future. Aside from efforts to improve the prevention of $A K I$, the use of PD will play a crucial role in low-income countries in treating the episodes of AKI that cannot be averted. Nevertheless, even in developed nations it is important to consider PD in AKI for certain patients, despite the availability of continuous hemodialysis.

\section{Hyperbranched Polyglycerol}

In 1923, the first PD trial was conducted by Ganter using a saline solution. Shortly after, in 1927, Heusser added dextrose to improve ultrafiltration making it the main osmotic agent in PD solutions that continues until today. Since the first commercially available solutions in 1959, little has changed. Amino acids are infrequently used instead of glucose, and lactate can be substituted with bicarbonate as a buffer, both options being used depending on clinician's preferences given the lack of consistent evidence for superiority of one over the other. Dextrose as the main osmotic agent is far from perfect, however. It is easily absorbed systemically leading to continuous glucose loading. In addition, glucose may have negative effects on peritoneal host defense. The introduction of icodextrin proved to be a valuable option for longer dwells, 


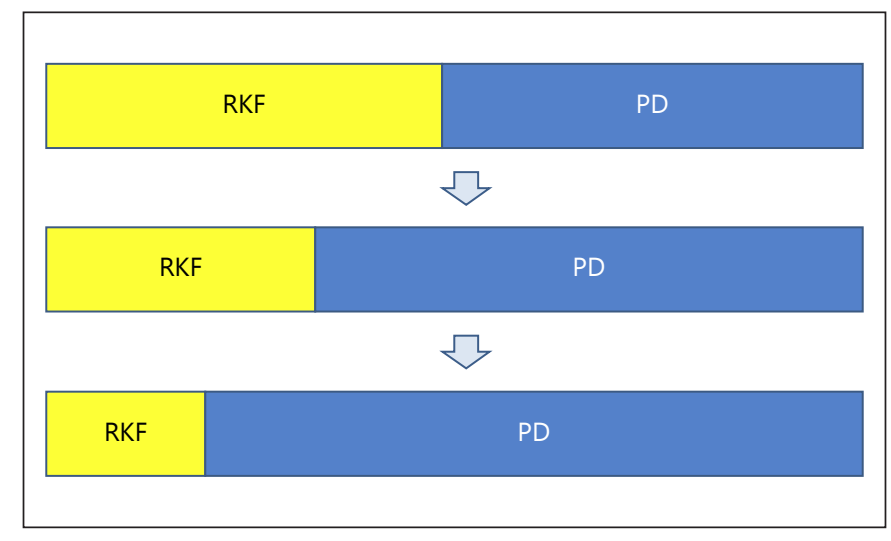

Fig. 2. Concept of incremental PD. As RKF decreases, the dose of $\mathrm{PD}$ is increased incrementally. RKF, residual kidney function; PD, peritoneal dialysis.

especially in rapid transporters, but its once-daily use makes it a supplement rather than an overall substitution for dextrose. Therefore, the search for other PD solutions continues.

An interesting new solute is hyperbranched polyglycerol (HPG). Theoretically, the molecular properties of HPG make it more efficient than glucose in producing ultrafiltration. Promising studies $[15,16]$ in rodents showed better preservation of UF and fewer toxic effects on the peritoneal membrane. However, studies in humans have not yet been conducted, and there are several questions remaining such as the ramifications of accumulation of HPG over time, or whether HPG molecules are metabolized in plasma and in the peritoneal cavity. Also, the long-term biocompatibility over years remains unclear, with the longest animal experiment using the solution for 3 months [15].

In summary, despite the real and theoretical risks of glucose, it remains the principal osmotic agent for now. HPG is a promising molecule for the future, but further research is needed before it can be used clinically.

\section{Incremental PD}

A considerable number of patients starting RRT have significant residual kidney function (RKF). The rationale of incremental PD (incrPD) is to treat symptoms of renal failure by a combination of dialysis and the RKF. With the further decline of kidney function, dialysis dosage is continually increased (Fig. 2). IncrPD was first used during the late 1990s but considered an early start of RRT before symptoms supervened $[17,18]$. When prescribing in-
$\mathrm{crPD}$, it is crucial to monitor RKF in order to increase PD when necessary. RKF can be monitored by 24 -h urine collection.

There are several advantages to this approach: first, given significant RKF $(3-10 \mathrm{~mL} / \mathrm{min})$ even a small amount of PD tends to improve uremic symptoms. Second, there is reduced burden on the patient with a less intensive regimen, giving the patient time to adjust to PD. Third, the glucose exposure of the peritoneum can be minimized from the beginning. Finally, there is evidence showing that incrPD might better conserve RKF [19]. Losing RKF not only leads to a reduction in the quality of life, but it is also an important prognostic factor [20].

Studies on incremental dialysis with a large number of patients are scarce. Sandrini et al. [19] included 105 patients and showed no difference in survival and peritonitis rate over a median time of 17 months when compared to standard PD (stPD; 3-5 exchanges in $24 \mathrm{~h}$ ). IncrPD was defined by one or 2 dwell times per day. Weekly peritoneal $\mathrm{Kt} / \mathrm{V}$ was expectedly lower with incrPD, but total weekly Kt/V targets were met in both groups. Strikingly, RKF remained stable in incrPD over the study period, whereas it decreased in stPD. Another study [21] showed that most patients starting on incrPD (defined as $\leq 3$ changes per day) stayed stable during the first 2 years. Interestingly, there was a reduction in the rate of renal function loss after initiation of incrPD showing that the described preservation of RKF in stPD is the same or even better with incrPD. In both these studies $[19,21]$, a significant cohort of patients were transplanted before any increase in the dose of PD was necessary.

In summary, the available literature shows at least non-inferior survival patterns compared to stPD, and suggests superiority for sustaining RKF. Considering the aforementioned advantages, IncrPD should therefore be considered in most patients with significant RRT $(>5 \mathrm{~mL} /$ $\min )$.

\section{Home Generation of PD Solutions}

Compared to $\mathrm{HD}, \mathrm{PD}$ is technically less challenging and therefore less costly, especially in developed countries. However, the delivery and storage of PD solutions are cumbersome and a burden on patients. Additionally, PD solution boxes weigh up to 30 pounds, being a potential problem to lift and carry, especially for older and weaker patients. While dialysate production at home is standard for home $\mathrm{HD}$, there is currently no technique 
available to do the same for PD solutions. This would be a big step forward, not only for patient's convenience but also by being more cost effective and by decreasing the carbon foot print for PD with delivery of solutions to the patient's home.

In May 2017, Baxter International Inc., announced [22] a new system for home production of sterile PD solution with the first patient planned to be on therapy in 2018 as part of a research trial. The solution generation system will be part of an automated PD system and is supposed to give physicians the option to prescribe different dextrose concentrations that can be changed at home by the patient or caregiver.

\section{PD in the Age of iPhone and Apps - Advances in Remote Monitoring}

Wearable technical devices like glasses, armbands and especially watches are a fast growing industrial market. Overall, there are 2 categories: consumer and non-consumer applications. Consumer applications are marketed for fitness, sport, fashion and gaming and make up 65\% of sold wearables, where the other $35 \%$ are non-consumer wearables that are made for health care and other areas like defense [23]. Studies estimate the market for nonconsumer products to grow from 3.2 billion in 2014 to 8.1 billion in 2018 [23]. Comparing those numbers to the estimated 22.1 billion in 2018 [23] for consumer products explains why currently most innovations are made in the consumer products area. Further, the threshold for a medical device or application to get regulatory approval is much higher than that for technical devices intended for recreational use.

Different aspects of wearable devices are of potential interest for physicians treating PD patients. Currently, biosensors on devices such as Apple's iWatch and the associated research kit software [24] are already able to monitor heart rate, sleep pattern, balance, gait and activity. Data on activity and sleeping patterns can give important information on patients' well-being and quality of life. Monitoring motion and balance has the potential of fall risk assessment, thereby preventing fall events, a major problem in older and frail patients. Another monitoring technology not yet integrated in commercially available devices is bioimpedance spectroscopy. Delano and Sodini [25] reported on developing a portable system with the ability to transmit data over Bluetooth to an iOS device and studies in HF and dialysis patients are ongoing.

In summary, while currently mostly used for recreational use, wearable devices in combination with sophisticated software have the potential to be a useful help in the medical field and improve patient care in the future.

\section{Conclusion}

The future lies in continuing to apply the knowledge we have but also to stay open to change. Since PD is different from $\mathrm{HD}$, it has unique advantages, and it is important to keep thinking about its use in special clinical settings.

\section{Disclosure Statement}

Dr. Bargman is a consultant for NxStage, Baxter Global, Keryx, Rockwell Medical and has received Speaker's Fees from DaVita Healthcare Partners, Baxter Canada and Janssen Ortho Canada. No potential conflict of interest was reported by Dr. Girsberger.

\section{References}

1 Wegner G: Chirurgische Bemerkungen über die Peritonealhöhle, mit besonderer Berücksichtigung der Ovariotomie. Arch Klin Chir 1877;20:51.

2 Mullens W, Abrahams Z, Francis GS, Sokos G, Taylor DO, Starling RC, et al: Importance of venous congestion for worsening of renal function in advanced decompensated heart failure. J Am Coll Cardiol 2009;53: 589-596.

3 Zemel D, Imholz AL, de Waart DR, Dinkla C, Struijk DG, Krediet RT: Appearance of tumor necrosis factor-alpha and soluble TNF-recep- tors I and II in peritoneal effluent of CAPD. Kidney Int 1994;46:1422-1430.

4 Selby NM, McIntyre CW: Peritoneal dialysis is not associated with myocardial stunning. Perit Dial Int 2011;31:27-33.

5 Selby NM, Lambie SH, Camici PG, Baker CS, McIntyre CW: Occurrence of regional left ventricular dysfunction in patients undergoing standard and biofeedback dialysis. Am J Kidney Dis 2006; 47:830-841.

6 Courivaud C, Kazory A, Crepin T, Azar R, Bresson-Vautrin C, Chalopin JM, et al: Peritoneal dialysis reduces the number of hospi- talization days in heart failure patients refractory to diuretics. Perit Dial Int 2014;34:100108.

7 Bertoli SV, Musetti C, Ciurlino D, Basile C, Galli E, Gambaro G, et al: Peritoneal ultrafiltration in refractory heart failure: a cohort study. Perit Dial Int 2014;34:64-70.

8 Núñez J, González M, Miñana G, GarciaRamón R, Sanchis J, Bodí V, et al: Continuous ambulatory peritoneal dialysis as a therapeutic alternative in patients with advanced congestive heart failure. Eur J Heart Fail 2012;14: 540-548. 
9 Gaião S, Finkelstein FO, de Cal M, Ronco C, Cruz DN: Acute kidney injury: are we biased against peritoneal dialysis? Perit Dial Int 2012;32:351-355.

10 Gabriel DP, Caramori JT, Martim LC, Barretti P, Balbi AL: High volume peritoneal dialysis vs daily hemodialysis: A randomized, controlled trial in patients with acute kidney injury. Kidney Int 2008;73:S87-S93.

11 Ponce D, Berbel MN, Abrão JM, Goes CR, Balbi AL: A randomized clinical trial of high volume peritoneal dialysis versus extended daily hemodialysis for acute kidney injury patients. Int Urol Nephrol 2013;45:869-878.

12 Chionh CY, Soni SS, Finkelstein FO, Ronco C, Cruz DN: Use of peritoneal dialysis in AKI: a systematic review. Clin J Am Soc Nephrol 2013;8:1649-1660.

13 Susantitaphong P, Cruz DN, Cerda J, Abulfaraj M, Alqahtani F, Koulouridis I, et al: World Incidence of AKI: a meta-analysis. Clin J Am Soc Nephrol 2013;8:1482-1493.

14 Mehta RL, Burdmann EA, Cerdá J, Feehally J, Finkelstein F, García-García G, et al: Recognition and management of acute kidney injury in the International Society of Nephrology 0by 25 Global Snapshot: a multinational crosssectional study. Lancet 2016;387:2017-2025.

15 Du C, Mendelson AA, Guan Q, Dairi G Chafeeva I, Da Roza G, et al: Hyperbranched polyglycerol is superior to glucose for longterm preservation of peritoneal membrane in a rat model of chronic peritoneal dialysis. J Transl Med 2016;14:338.

16 Mendelson AA, Guan Q, Chafeeva I, da Roza GA, Kizhakkedathu JN, Du C: Hyperbranched polyglycerol is an efficacious and biocompatible novel osmotic agent in a rodent model of peritoneal dialysis. Perit Dial Int 2013;33:15-27.

17 Nolph KD: Rationale for early incremental dialysis with continuous ambulatory peritoneal dialysis. Nephrol Dial Transplant 1998; 13(suppl 6):117-119.

18 Mehrotra R, Nolph KD, Gotch F: Early initiation of chronic dialysis: role of incremental dialysis. Perit Dial Int 1997;17:426430.

19 Sandrini M, Vizzardi V, Valerio F, Ravera S, Manili L, Zubani R, et al: Incremental perito- neal dialysis: a 10 year single-centre experience. J Nephrol 2016;29:871-879.

20 Wang AY, Lai KN: The importance of residual renal function in dialysis patients. Kidney Int 2006;69:1726-1732.

21 Borràs Sans $M$, Chacón Camacho A, Cerdá Vilaplana C, Usón Nuño A, Fernández E: Diálisis peritoneal incremental: resultados clínicos y preservación de la función renal residual. Nefrología 2016;36: 299-303.

22 Baxter International Inc.: Baxter Achieves Regulatory Milestone for New Peritoneal Dialysis Technology, 2017.

23 Salah H, MacIntosh E, Rajakulendran N: Wearable Tech: Leveraging Canadian Innovation to Improve Health. 2014. https://www. marsdd.com/wp-content/uploads/2015/02/ MaRSReport-WearableTech.pdf (cited August 28, 2017).

24 https://www.apple.com/researchkit/.

25 Delano MK, Sodini CG: A Portable Bioimpedance Spectroscopy Measurement System for Management of Congestive Heart Failure, 2016 